ARTÍ́CULO

\title{
Variabilidad espacio-temporal del fitoplancton de la ensenada Mackellar, Bahía Almirantazgo, Isla Rey Jorge, Antártida, durante el verano austral 2012/2013
}

Spatial and temporal variability of the phytoplankton in the Mackellar inlet, Admiralty Bay, King George Island, Antarctic, during summer 2012/2013

\author{
Maribel Baylón ${ }^{1}$, David U. Hernández-Becerril2*, Aldo Indacochea ${ }^{3}$ y Sara Purca ${ }^{4}$ \\ 'Laboratorio de Ecología Acuática, Facultad de Ciencias Biológicas, Universidad Nacional Mayor de San Marcos, Lima, Perú \\ ${ }^{2}$ Instituto de Ciencias del Mar y Limnología, Universidad Nacional Autónoma de México, Ciudad Universitaria, Ciudad de México, México \\ ${ }^{3}$ Laboratorio de Ecología Marina, Facultad de Ciencias Veterinarias y Biológicas, Universidad Científica del Sur, Lima, Perú \\ ${ }^{4}$ Área de Investigaciones Marino Costeras, Dirección de Investigaciones en Acuicultura, Instituto del Mar del Perú - IMARPE, Lima, Perú \\ *Autor de correspondencia: dhernand@cmarl.unam.mx
}

\begin{abstract}
The Antarctic ecosystem is one of the most sensitive world regions to the climate change, and exhibits a wide environmental variability that modifies the temporal and spatial patterns of the phytoplankton structure. The phytoplankton community and its spatial and temporal variability of Mackellar inlet- Admiralty bay, Antarctic, were studied in two austral summers (years 2012 and 2013). Samplings were done in 11 fixed stations at three depths $(0,10$ and $20 \mathrm{~m})$. Antarctic Surface Water mass was identified in the surface layer at the study area. Phytoplankton composition in the water column consisted in 40 diatoms taxa, 6 dinoflagellate taxa and various species of nanoflagellates $(<20 \mu \mathrm{m})$. Nanoflagellates from the Mackellar inlet included Prasinophytes, Cryptophytes and Haptophytes species. Diatoms of the microplankton fraction $(20-200 \mu \mathrm{m})$ and nanoflagellates $(<20 \mu \mathrm{m})$ were in alternation during the period of this study: by summer 2012 nanoflagellates were more abundant ( $86 \%$ ), with diatom species of the genus Thalassiosira (11\%), and by summer 2013 phytoplankton abundance was dominated by microplanktonic diatoms such as Thalassiosira delicatula (36\%), Pseudonitzschia group delicatissima (9\%), Porosira glacialis (6\%), the athecate dinoflagellate Gymnodinium sp. (19\%) and nanoflagellates (20\%). Cell densities were lower in summer 2012 compared with those in summer 2013, where a diatom bloom occurred. Phytoplankton diversity was relatively low $\left(<1.97\right.$ bits $\cdot$ cell $\left.^{-1}\right)$ in both summers. The main differences between the two sampling periods were the dominance of microplanktonic diatoms and nanoflagellates, which were probably caused by the decrease in salinity and increase in subsurface dissolved oxygen in the study area produced by ice melting in summer 2012 and 2013.
\end{abstract}

Key words: Antarctic, diatoms, microplankton, nanoflagellates, variability

Resumen.- El ecosistema antártico es una de las regiones del mundo más sensibles al cambio climático, y presenta amplia variabilidad ambiental que modifican los patrones espaciales y temporales de la estructura fitoplanctónica. Se estudió la comunidad del fitoplancton y su variabilidad espacial y temporal de la ensenada Mackellar-bahía Almirantazgo, Antártida, en los veranos australes de 2012 y 2013. Se hicieron muestreos de agua para fitoplancton y variables ambientales en 11 estaciones a tres profundidades (0,10 y $20 \mathrm{~m})$. La masa de Agua Superficial Antártica de la zona de estudio fue reconocida. La composición total del fitoplancton en la columna de agua incluyó 40 taxones de diatomeas, 6 taxones de dinoflagelados y varias especies de nanoflagelados $(<20 \mu \mathrm{m})$. Los nanoflagelados pertenecen a Prasinophyceae, Cryptophyta y Haptophyta. La alternancia entre diatomeas del microplancton (20-200 $\mu \mathrm{m})$ y nanoflagelados $(<20$ $\mu \mathrm{m})$ fue evidente durante el período de estudio: en el verano 2012 el grupo de los nanoflagelados fue más abundante (86\%), con especies de diatomeas del género Thalassiosira sp. (11\%), y para el verano 2013 los nanoflagelados disminuyeron considerablemente (20\%), la abundancia del fitoplancton estuvo dominada por diatomeas como Thalassiosira delicatula (36\%), Pseudo-nitzschia grupo delicatissima (9\%), Porosira glacialis (6\%), y el dinoflagelado Gymnodinium sp. (19\%). La densidad celular en el verano 2012 fue menor, en comparación con el verano 2013, donde ocurrió una floración de diatomeas. La diversidad del fitoplancton fue baja (<1,97 bits·célula ${ }^{-1}$ ) en ambos veranos. Las principales diferencias fueron la dominancia de diatomeas del microplancton y nanoflagelados, las cuales probablemente fueron causadas por la disminución de la salinidad y el aumento de oxígeno superficial observado en la Antártida, producto del deshielo ocurrido durante los veranos 2012 y 2013.

Palabras clave: Antártida, diatomeas, microplancton, nanoflagelados, variabilidad

\section{INTRODUCCIÓN}

El ecosistema antártico presenta un especial interés científico por sus características particulares, ya que mantienen altas concentraciones de nutrientes inorgánicos, que son utilizados por el fitoplancton autótrofo, base de la cadena alimenticia (Torres et al. 2006), que incluye más de 200 especies de crustáceos, resaltando el "krill" (pequeños crustáceos de alto contenido proteico que debido a su abundancia llega a teñir de un color rojizo algunas zonas marinas), así como peces, focas, lobos marinos, aves y ballenas.

Durante la primavera y verano austral se desarrolla la mayor producción planctónica, siendo consecuencia directa del aporte de nutrientes procedentes del deshielo y 
del incremento de la intensidad luminosa, que en latitudes altas pasa de ser prácticamente nula durante el invierno a durar casi las $24 \mathrm{~h}$ del día en primavera (Gili et al. 2000). A estas floraciones de productores primarios suceden las de consumidores primarios: zooplancton herbívoro, como el krill. En muchos casos, los consumidores no agotan la producción primaria, generada en poco tiempo y a gran escala. Una vez terminado el verano y durante el prolongado invierno austral, la oscuridad y la escasez de nutrientes frenan la producción planctónica y, por ende, cortan el suministro vertical de alimento (Gili et al. 2000).

En las últimas décadas, Perú ha registrado escasas publicaciones científicas sobre la abundancia del plancton en las Islas Shetland del Sur, Antártida. En contraste, recientemente se ha producido un incremento de publicaciones sobre estudios del plancton de la Antártida debido al rol ecológico que la producción primaria y secundaria podrían tener en los polos en un escenario de calentamiento global (Sayed 2005, Bonicelli et al. 2008). Las Campañas Científicas del Perú a la Antártida (ANTAR) se vienen realizando desde 1988 en la ensenada Mackellar y la bahía Almirantazgo, Estrecho de Bransfield, la Isla Rey Jorge (perteneciente a las Islas Shetland del Sur) y alrededores de la Isla Elefante, ambas vinculadas a la Península Antártica (PA). Sánchez \& Villanueva (2001) mostraron cambios marcados en la composición taxonómica del fitoplancton, la cual se caracterizó por una alternancia en la especie dominante dada por las diatomeas o el nanoplancton. En particular, los estudios relacionados con la composición del fitoplancton en el sur de las Islas Shetland y los alrededores de la PA han sido relativamente abundantes (García-Muñoz et al. 2013). Recientemente en la bahía Almirantazgo se ha reportado una dominancia de nanoflagelados en número y biomasa sobre diatomeas del microplancton, en las cercanías de la zona costera de la estación Polaca Henryk Arctowski, donde se encontró una tendencia de disminución de dichas diatomeas en la contribución total, de 44\% entre 1996-1998 a 5\% entre 2003-2005 (Wasilowska et al. 2015). Adicionalmente, Vanzan et al. (2015) mostraron una alternancia estacional entre nanoplancton y picoplancton asociado al sistema de corrientes de mesoescala y el pastoreo estacional, donde sugieren que a futuro se debe desarrollar trabajos para comunidades $<20 \mu \mathrm{m}$ para estudiar los efectos en la trama trófica microbiana y sus posibles efectos ambientales en altos niveles tróficos de las zonas costeras de la bahía Almirantazgo.

La bahía Almirantazgo se ha convertido en un sitio de actividades humanas de diversidad creciente que continuamente se están multiplicando y más complejas. Durante los últimos 30 años se han emplazado más estaciones en la zona, que han ido creciendo, y el número de visitantes ha aumentado cada año, pasando de algunos cientos a más de 3.000 (Secretaría del Trabajo Antártica 1996). La Península Antártica (PA) es una de las regiones del mundo más sensibles al cambio climático (Turner et al.2005), sin embargo, las consecuencias de estos cambios todavía no se entienden completamente. Los cambios ambientales en la región de la PA han estado modificando el espacio y los patrones temporales de la biomasa y composición del fitoplancton (Garibotti et al. 2005). En la cabecera de la bahía Almirantazgo, el casquete glacial desemboca en tres ensenadas: Ezcurra, Mackellar y Martel, y específicamente el área de la ensenada Mackellar, es una zona importante por ser un hábitat protegido y somero, y en la actualidad no se han publicado trabajos sobre el fitoplancton en esa zona, por lo que es significativo realizar estudios y complementar la información de microalgas planctónicas para la bahía Almirantazgo, Isla Rey Jorge, Antártida.

Los primeros estudios de fitoplancton en la bahía Almirantazgo fueron dados por Kopczyńska (1980, 1981, 1996), los cuales ofrecen información de la composición, distribución vertical y estacional del fitoplancton de la Ensenada Ezcurra, brindados por la estación Polaca Arctowski. La comunidad fitoplanctónica en la bahía Almirantazgo mostró una relación directa en los picos de floraciones algales con las condiciones hidrográficas y atmosféricas (Kopczyńska 1981). Así por bastante tiempo se ha planteado que la sucesión y los picos de fitoplancton en el océano sur son altamente influenciadas por las condiciones oceanográficas, tales como las corrientes, estabilidad de las aguas superficiales, recesión y duración del hielo (Hasle 1969, Steyeart 1973). Estudios de hidrografía extensiva en la bahía Almirantazgo han documentado una gran variabilidad de factores locales, tales como la alta velocidad de las corrientes, profundidad de las capas de mezcla y la influencia de aguas del Estrecho de Bransfield (Pruszak 1980, Rakusa-Suszczewski 1980, 1993; Szafrański \& Lipski 1982, Catewicz 1984). La influencia de agua dulce de la tierra (Rakusa-Suszczewski 1996), fuertes vientos en caída y la morfología de la cuenca de la bahía (Rakusa-Suszczewski \& Kidawa 1997, Robakiewicz \& Rakusa-Suszczewski 1999) pueden también afectar considerablemente las poblaciones de plancton.

Por lo anterior, el objetivo de la presente investigación fue identificar las especies fitoplanctónicas de la ensenada Mackellar-bahía Almirantazgo, Antártida y estudiar sus variaciones temporales (durante dos veranos) espaciales y verticales en la columna de agua (a 3 profundidades), junto con evaluar las posibles relaciones entre variables ambientales sobre la estructura del fitoplancton. 


\section{MATERIALES Y MÉTODOS}

\section{ÁREA DE ESTUDIO}

El área de estudio está localizada en la ensenada Mackellarbahía Almirantazgo (BA). La BA es la bahía más grande de la Isla Rey Jorge, Islas Shetland del Sur, cubriendo un área de $120 \mathrm{~km}^{2}$. Se encuentra entre los $62^{\circ} 04^{\prime}-62^{\circ} 14^{\prime} \mathrm{S}$ y $58^{\circ} 14^{\prime}-58^{\circ} 38^{\prime} \mathrm{W}$ (Fig. 1), a $750 \mathrm{~km}$ al sur-este de América del Sur (Fig. 1B y C).

El estudio abarcó toda el área de la ensenada Mackellar sobre la cual se establecieron 11 estaciones de muestreo. Las estaciones E01, E02, E03 y E04 están situadas en el primer transecto de la ensenada, colindante a los glaciales, mientras que las estaciones E05, E06, E07 y E08 están en el segundo transecto; cabe mencionar que la estación E05 está ubicada cerca a la Estación Científica Antártica y las estaciones E09, E10 y E11 en la entrada de la ensenada
(Fig. 1A). La localización geográfica de cada una de las estaciones se muestra en las Tablas 1 y 2.

\section{Procedimiento de MUeStreo}

Los muestreos fueron realizados del 20 al 22 de enero del verano austral 2012 (ANTAR XX) y del 2 al 6 de febrero del verano austral 2013 (ANTAR XXI). El trabajo se realizó a bordo de una embarcación tipo Zodiac ${ }^{\circledR}$. Se colectaron muestras de agua (botellas Niskin de $5 \mathrm{~L}$ de capacidad) a profundidades de 0,10 y $20 \mathrm{~m}$ de profundidad, para la determinación cuantitativa de fitoplancton. Las muestras obtenidas se fijaron con formalina hasta tener una concentración final del $2 \%$. Adicionalmente se midieron las variables ambientales de la columna de agua: temperatura, salinidad, oxígeno y $\mathrm{pH}$, con ayuda de una sonda multiparámetro de campo HANNA® HI 9828.

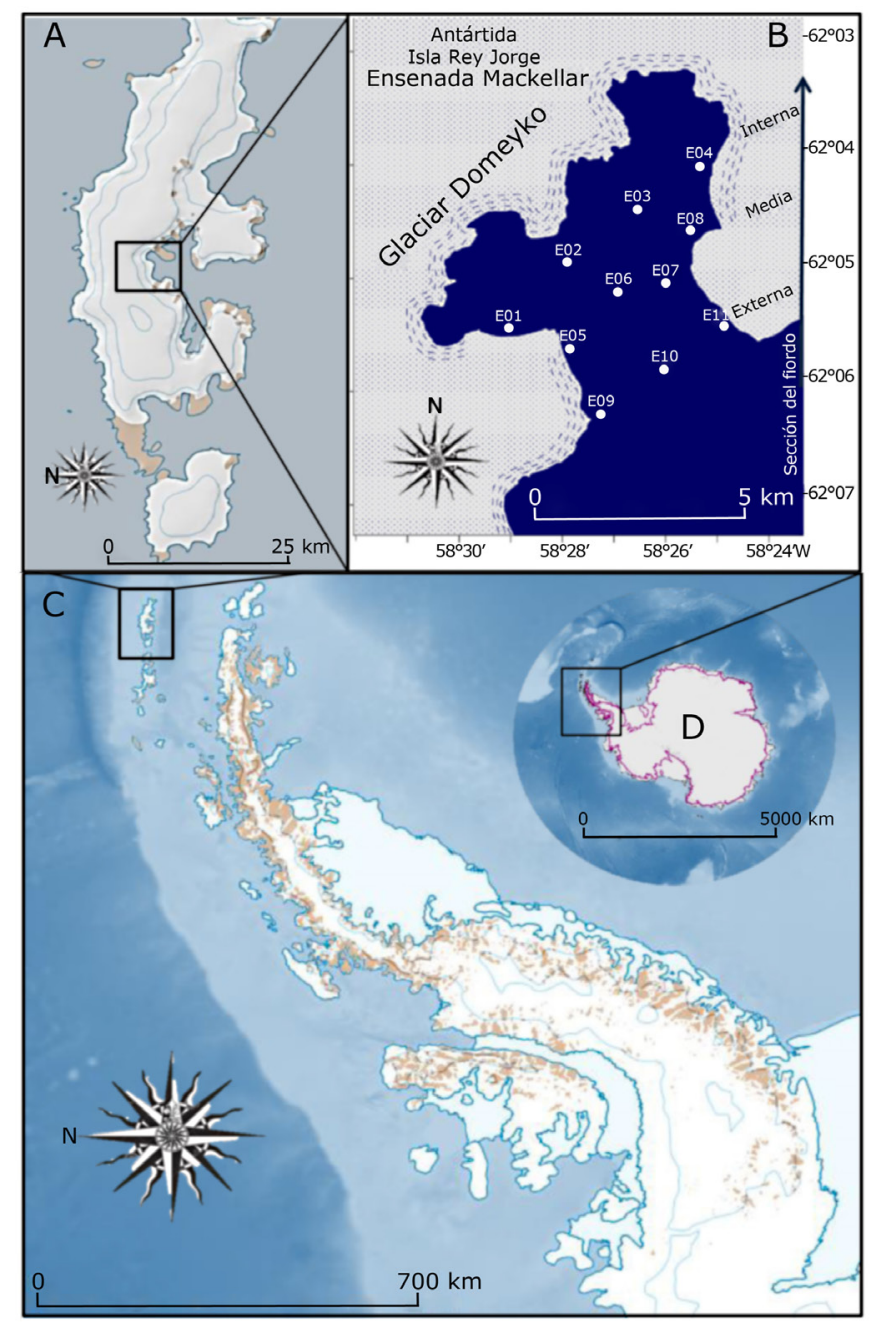

Figura 1. Mapa de ubicación de la ensenada Mackellar-Bahía Almirantazgo. A) Ensenada Mackellar, B) Estaciones de muestreo de la ensenada Mackellar, C) Isla Rey Jorge, D) Antártida / Map of the Mackellar inlet, Admiralty Bay. A) Mackellar inlet, B) Sampling stations at the Mackellar inlet, C) King George Island, D) Antarctic continent 
Tabla 1. Ubicación de las estaciones biológicas y valores de abundancia de diatomeas, dinoflagelados y nanoflagelados a tres profundidades durante la Campaña Científica ANTAR XX, verano 2012 / Location of the biological stations and values of abundance for diatoms, dinoflagellates and nanoflagellates at three depths during the oceanographic cruise ANTAR XX, summer 2012

\begin{tabular}{|c|c|c|c|c|c|c|c|c|c|c|c|}
\hline \multirow[t]{2}{*}{ Estación } & \multirow[t]{2}{*}{ Latitud sur } & \multirow{2}{*}{$\begin{array}{l}\text { Longitud } \\
\text { oeste }\end{array}$} & \multicolumn{3}{|c|}{$\begin{array}{l}\text { Abundancia diatomeas } \\
\left(\text { células } \cdot \mathrm{L}^{-1}\right)\end{array}$} & \multicolumn{3}{|c|}{$\begin{array}{l}\text { Abundancia dinoflagelados } \\
\left.\text { (células } \cdot \mathrm{L}^{-1}\right)\end{array}$} & \multicolumn{3}{|c|}{$\begin{array}{l}\text { Abundancia nanoflagelados } \\
\qquad\left(\text { células } \cdot \mathrm{L}^{-1}\right)\end{array}$} \\
\hline & & & $0 \mathrm{~m}$ & $10 \mathrm{~m}$ & $20 \mathrm{~m}$ & $0 \mathrm{~m}$ & $10 \mathrm{~m}$ & $20 \mathrm{~m}$ & $0 \mathrm{~m}$ & $10 \mathrm{~m}$ & $20 \mathrm{~m}$ \\
\hline 1 & $62^{\circ} 05^{\prime} 7.4^{\prime \prime}$ & $58^{\circ} 29^{\prime} 0.5^{\prime \prime}$ & 25.911 & 6.780 & 17.706 & 580 & 675 & 562 & 20.011 & 9.422 & 15.711 \\
\hline 2 & $62^{\circ} 04^{\prime} 14.05^{\prime \prime}$ & $58^{\circ} 27^{\prime} 14.9^{\prime \prime}$ & 13.520 & 17.524 & 20.624 & 909 & 660 & 2.178 & 554 & 12.320 & 17.730 \\
\hline 3 & $62^{\circ} 04^{\prime} 3.6^{\prime \prime}$ & $58^{\circ} 26^{\prime} 0.5^{\prime \prime}$ & 2.920 & 8.400 & 7.900 & 20 & 380 & 1.295 & 0 & 34.297 & 14.745 \\
\hline 4 & $62^{\circ} 04^{\prime} 0.3^{\prime \prime}$ & $58^{\circ} 25^{\prime} 5.1^{\prime \prime}$ & 9.195 & 3.840 & 7.820 & 0 & 100 & 400 & 1.955 & 5.237 & 5.160 \\
\hline 5 & $62^{\circ} 05^{\prime} 39^{\prime \prime}$ & $58^{\circ} 27^{\prime} 53.9^{\prime \prime}$ & 4.700 & 5.000 & 3.860 & 120 & 260 & 1.109 & 18.611 & 24.527 & 53.886 \\
\hline 6 & $62^{\circ} 05^{\prime} 2.1^{\prime \prime}$ & $58^{\circ} 26^{\prime} 15.4^{\prime \prime}$ & 4.980 & 4.820 & 16.060 & 80 & 1.249 & 1.189 & 2.729 & 17.847 & 35.608 \\
\hline 7 & $62^{\circ} 05^{\prime} 0.3^{\prime \prime}$ & $58^{\circ} 25^{\prime} 16.5^{\prime \prime}$ & 2.120 & 6.640 & 15.858 & 40 & 540 & 420 & 2.690 & 16.000 & 32.176 \\
\hline 8 & $62^{\circ} 04^{\prime} 33.1^{\prime \prime}$ & $58^{\circ} 25^{\prime} 29.4^{\prime \prime}$ & 460 & 860 & 660 & 0 & 140 & 260 & 31.650 & 126.991 & 366.551 \\
\hline 9 & $62^{\circ} 06^{\prime} 09.6^{\prime \prime}$ & $58^{\circ} 27^{\prime} 17^{\prime \prime}$ & 460 & 980 & 740 & 40 & 700 & 60 & 408.510 & 163.507 & 140.330 \\
\hline 10 & $62^{\circ} 06^{\prime} 10^{\prime \prime}$ & $58^{\circ} 27^{\prime} 04.2^{\prime \prime}$ & 1.900 & 1.940 & 3.340 & 0 & 535 & 180 & 2.309 & 7.800 & 7.986 \\
\hline 11 & $62^{\circ} 05^{\prime} 24.8^{\prime \prime}$ & $58^{\circ} 24^{\prime} 53.5^{\prime \prime}$ & 2.540 & 4.140 & 1.360 & 60 & 160 & 40 & 2.009 & 10.447 & 2.315 \\
\hline Total & & & 68.706 & 60.924 & 95.928 & 1.849 & 5.399 & 7.693 & 491.028 & 428.395 & 692.198 \\
\hline
\end{tabular}

Tabla 2. Ubicación de las estaciones biológicas y valores de abundancia de diatomeas, dinoflagelados y nanoflagelados a tres profundidades durante la Campaña Científica ANTAR XXI, verano 2013 / Location of the biological stations and values of abundance for diatoms, dinoflagellates and nanoflagellates at three depths during the oceanographic cruise ANTAR XXI, summer 2013

\begin{tabular}{|c|c|c|c|c|c|c|c|c|c|c|c|}
\hline \multirow[t]{2}{*}{ Estación } & \multirow[t]{2}{*}{ Latitud sur } & \multirow{2}{*}{$\begin{array}{l}\text { Longitud } \\
\text { oeste }\end{array}$} & \multicolumn{3}{|c|}{$\begin{array}{l}\text { Abundancia diatomeas } \\
\left(\text { células } \cdot \mathrm{L}^{-1}\right)\end{array}$} & \multicolumn{3}{|c|}{$\begin{array}{l}\text { Abundancia dinoflagelados } \\
\text { (células } \cdot \mathrm{L}^{-1} \text { ) }\end{array}$} & \multicolumn{3}{|c|}{$\begin{array}{c}\text { Abundancia nanoflagelados } \\
\text { (células } \cdot \mathrm{L}^{-1} \text { ) }\end{array}$} \\
\hline & & & $0 \mathrm{~m}$ & $10 \mathrm{~m}$ & $20 \mathrm{~m}$ & $0 \mathrm{~m}$ & $10 \mathrm{~m}$ & $20 \mathrm{~m}$ & $0 \mathrm{~m}$ & $10 \mathrm{~m}$ & $20 \mathrm{~m}$ \\
\hline 1 & $62^{\circ} 05^{\prime} 7.4^{\prime \prime}$ & $58^{\circ} 29^{\prime} 0.5^{\prime \prime}$ & 4.560 & 8.729 & 6.602 & 220 & 20 & 120 & 909 & 909 & 989 \\
\hline 2 & $62^{\circ} 04^{\prime} 14.05^{\prime \prime}$ & $58^{\circ} 27^{\prime} 14.9^{\prime \prime}$ & 2.320 & 6.540 & 7.780 & 0 & 100 & 60 & 0 & 909 & 3.182 \\
\hline 3 & $62^{\circ} 04^{\prime} 3.6^{\prime \prime}$ & $58^{\circ} 26^{\prime} 0.5^{\prime \prime}$ & 5.680 & 8.449 & 9.860 & 0 & 0 & 1.464 & 2.273 & 4.091 & 0 \\
\hline 4 & $62^{\circ} 04^{\prime} 0.3^{\prime \prime}$ & $58^{\circ} 25^{\prime} 5.1^{\prime \prime}$ & 2.240 & 7.520 & 9.840 & 0 & 80 & 40 & 0 & 3.636 & 0 \\
\hline 5 & $62^{\circ} 05^{\prime} 39^{\prime \prime}$ & $58^{\circ} 27^{\prime} 53.9^{\prime \prime}$ & 5.520 & 5.940 & 13.160 & 20 & 120 & 60 & 1.364 & 5.000 & 909 \\
\hline 6 & $62^{\circ} 05^{\prime} 2.1^{\prime \prime}$ & $58^{\circ} 26^{\prime} 15.4^{\prime \prime}$ & 5.349 & 3.580 & 9.260 & 20 & 60 & 40 & 909 & 1.818 & 6.364 \\
\hline 7 & $62^{\circ} 05^{\prime} 0.3^{\prime \prime}$ & $58^{\circ} 25^{\prime} 16.5^{\prime \prime}$ & 2.960 & 7.280 & 13.240 & 20 & 200 & 120 & 455 & 1.818 & 5.454 \\
\hline 8 & $62^{\circ} 04^{\prime} 33.1^{\prime \prime}$ & $58^{\circ} 25^{\prime} 29.4^{\prime \prime}$ & 3.260 & 4.340 & 11.760 & 0 & 100 & 0 & 909 & 11.818 & 5.000 \\
\hline 9 & $62^{\circ} 06^{\prime} 09.6^{\prime \prime}$ & $58^{\circ} 27^{\prime} 17^{\prime \prime}$ & 16.200 & 3.200 & 8.820 & 0 & 455 & 495 & 3.182 & 1.818 & 4.091 \\
\hline 10 & $62^{\circ} 06^{\prime} 10^{\prime \prime}$ & $58^{\circ} 27^{\prime} 04.2^{\prime \prime}$ & 5.680 & 2.860 & 7.560 & 26.363 & 8.580 & 780 & 909 & 6.818 & 2.727 \\
\hline 11 & $62^{\circ} 05^{\prime} 24.8^{\prime \prime}$ & $58^{\circ} 24^{\prime} 53.5^{\prime \prime}$ & 5.822 & 7.360 & 8.220 & 0 & 320 & 39.857 & 0 & 5.909 & 0 \\
\hline Total & & & 59.591 & 65.798 & 106.102 & 26.643 & 10.035 & 43.036 & 10.910 & 44.544 & 28.716 \\
\hline
\end{tabular}

\section{Procesamiento de muestras}

El análisis se realizó en el Laboratorio de Ecología Acuática y Laboratorio de Hidrobiología y Pesquería de la Facultad de Ciencias Biológicas de la Universidad Nacional Mayor de San Marcos. El análisis de fitoplancton se realizó bajo un microscopio invertido marca Leica, aplicando la metodología de Utermöhl (Lund et al. 1958), que consistió en homogeneizar las muestras suavemente y sedimentarlas en cámaras cilíndricas de $50 \mathrm{~mL}$, por un tiempo no menor de $48 \mathrm{~h}$. Para el conteo de las especies menores de $20 \mu \mathrm{m}$ y de las especies muy abundantes se emplearon aumentos de 40X, en un área de $86,75 \mathrm{~mm}^{2}$. Los organismos mayores a 20 $\mu \mathrm{m}$ y poco abundantes fueron contados en toda la cámara a 20X. En cada muestra se contaron al menos 100 células a lo largo de los transectos de la cámara elegidos aleatoriamente.
Los resultados obtenidos se dieron en número de células por litro $\left(\mathrm{N}^{\circ}\right.$ cél $\left.\cdot \mathrm{L}^{-1}\right)$.

Las diatomeas fueron identificadas hasta el nivel de género o especie. Debido a que no fue posible determinar todos los organismos encontrados, como el caso del grupo de nanoflagelados atecados $(<20 \mu \mathrm{m})$ fueron identificados hasta el nivel de clase. Los nanoflagelados de la ensenada Mackellar incluyen especies de Prasinophyceae, Cryptophyta y Haptophyta; los dinoflagelados no se incluyeron dentro del grupo de los nanoflagelados. Para la identificación de las microalgas se emplearon las obras de Frenguelli (1943), Balech (1944), Frenguelli \& Orlando (1958), Balech (1976), Sournia et al. (1979), Priddle \& Fryxell (1985), Medlin \& Priddle (1990), Round et al. (1990), Hasle \& Syvertsen (1997). 


\section{Análisis eSTAdístico}

Se realizó el análisis estadístico en relación a la naturaleza de los datos y se necesitó el uso de herramientas de comparación no paramétricas. Para los aspectos cuantitativos de la estructura comunitaria se empleó la abundancia y número de especies. La abundancia en cada estación de muestreo se empleó para calcular la diversidad empleando el índice de Shannon-Weaver (1963) usando $\log _{2}$. Los resultados se expresan en bits·células ${ }^{-1}$. Los análisis univariados y multivariados se realizaron usando el programa PAST versión 3.14 (Hammer et al. 2001, 2013) y PRIMER, versión 6 (Clarke \& Gorley 2006).

\section{MÉTOdos MULTIVARIADOS}

Para la identificación de las principales asociaciones florísticas se realizó el análisis de clasificación jerárquica (cluster) en base a una matriz de similitud entre muestras (previa integración de los datos de 0,10 y 20 m) elaborada a partir de los índices de Bray-Curtis y se expresó en un dendrograma usando el método de agrupamiento UPGMA. Para estos análisis, los datos de abundancia fueron transformados con $\log (\mathrm{x}+1)$. Se empleó el test de ANOSIM también con los datos de abundancia estandarizados $\log (\mathrm{x}+1)$ para evaluar las diferencias entre las profundidades $(0,10$ y $20 \mathrm{~m})$. Posteriormente se realizó el procedimiento SIMPER para identificar los taxones que más contribuyeron con la disimilitud de Bray Curtis entre los estratos y los veranos estudiados.

Para determinar el grado de correspondencia de las variables biológicas (grupos fitoplanctónicos) y las variables abióticas, se empleó el Análisis de Correspondencia Canónica (ACC).

\section{ANÁLISIS OCEANOGRÁFICO}

Adicionalmente se utilizó información procedente de 1.807 estaciones entre los años 1970 al 2000 de la base de datos oceanográfica mundial (WOD09 2016) ${ }^{1}$. Para el análisis de las masas de agua durante los veranos se utilizó el programa Ocean Data View (Schlitzer 2016) ${ }^{2}$, lo cual comprende los alrededores de las Islas del Sur Shetland y la Península Antártida.

\section{RESUltados}

\section{Hidrografía Y CONDICIONES OCEANOGRÁFICAS}

En el verano 2012, la temperatura superficial del mar (TSM) presentó un valor promedio de $1,63 \pm 0,22{ }^{\circ} \mathrm{C}$, mientras que en el verano 2013 la TSM presentó un valor promedio de $1,14 \pm 0,41{ }^{\circ} \mathrm{C}$ (Tabla 3).

Para el verano 2012, la salinidad promedio superficial del mar (SSM) fue de 34, para los $10 \mathrm{~m}$ de profundidad hubo un valor promedio de 34,43 , y para los $20 \mathrm{~m}$ de profundidad hubo un valor promedio de 34,51. Se evidenció una variación mínima de la salinidad promedio entre estratos. Para el verano del 2013, la SSM presentó un valor promedio de 33,69 , a $10 \mathrm{~m}$ de profundidad el valor promedio fue de 33,94 , mientras que a $20 \mathrm{~m}$ el promedio fue de 34,04 . Se evidenció un aumento de la salinidad promedio a $20 \mathrm{~m}$ de profundidad (Tabla 3).

El oxígeno disuelto y $\mathrm{pH}$ tuvieron muy pequeñas variaciones a lo largo de la ensenada. En la Tabla 3 se muestran las variables ambientales con sus respectivos valores mínimos, máximos y promedios con relación a la profundidad.

Las masas de agua promedio durante los veranos entre 1970 a 2000, según la base de datos mundial (1.807 estaciones) presentó Aguas Superficiales Antárticas y costeras entre $0-2{ }^{\circ} \mathrm{C}$ y salinidad de 34 , características de fiordos cerca de las Islas Shetland del Sur, mientras que Aguas Intermedias Antárticas se presentaron entre los 100 a 200 m. Aguas Antárticas de Fondo se presentaron a partir de los $500 \mathrm{~m}$ (Fig. 2).

Las masas de agua promedio durante los veranos del 2000 (WOD09 2016) ${ }^{1}$, indicaron Aguas Superficiales Antárticas y costeras entre $0-2^{\circ} \mathrm{C}$ y salinidad de 34 , y Aguas Intermedias Antárticas entre los 200 a 300 m. Se identificaron Aguas Antárticas de Fondo a partir de $\operatorname{los} 500$ m, a diferencia de los promedios totales de temperatura, durante 2000 se presentaron núcleos de hasta $3{ }^{\circ} \mathrm{C}$ al oeste de las Islas Shetland del Sur (Fig. 3).

${ }^{1}$ WOD09. 2016. Data Sets \& Products. World Ocean Database 2009. NOAA National Centers for Environmental Information - NCEI, Maryland Office, Website Survey <https://www.nodc.noaa.gov/OC5/WOD09/pr_wod09.htmll> ${ }^{2}$ Schlitzer R. 2016. Ocean Data View. <https://odv.awi.de> 
Tabla 3. Variables ambientales con sus valores mínimos, máximos, promedios con relación a la profundidad $(\mathrm{m})$ de los veranos 2012 y 2013 / Environmental variables with their minima, maxima and average values with respect to depth $(\mathrm{m})$ for the summers of 2012 and 2013

\begin{tabular}{lccccccccc}
\hline & \multirow{2}{*}{$\begin{array}{c}\text { Prof. } \\
(\mathrm{m})\end{array}$} & \multicolumn{2}{c}{ Temperatura $\left({ }^{\circ} \mathrm{C}\right)$} & \multicolumn{2}{c}{ Salinidad } & \multicolumn{2}{c}{ Oxígeno $\left(\mathrm{mL} \cdot \mathrm{L}^{-1}\right)$} & \multicolumn{2}{c}{$\mathrm{pH}$} \\
\cline { 3 - 11 } & 2012 & 2013 & 2012 & 2013 & 2012 & 2013 & 2012 & 2013 \\
\hline Min & 0 & 1,41 & 0,69 & 32,82 & 33,46 & 7,52 & 10,24 & 7,88 & 9,24 \\
Max & 0 & 1,84 & 1,51 & 34,47 & 34,04 & 9,80 & 12,70 & 9,49 & 10,01 \\
Prom & 0 & 1,63 & 1,14 & 33,99 & 33,69 & 8,54 & 11,10 & 8,47 & 9,80 \\
Min & 10 & 1,38 & 0,72 & 34,02 & 33,83 & 7,37 & 9,52 & 7,89 & 9,17 \\
Max & 10 & 1,56 & 1,14 & 34,70 & 34,02 & 9,77 & 11,30 & 9,42 & 9,92 \\
Prom & 10 & 1,49 & 1,01 & 34,43 & 33,94 & 8,27 & 10,44 & 8,52 & 9,78 \\
Min & 20 & 1,33 & 0,77 & 34,36 & 33,94 & 7,70 & 9,04 & 7,84 & 9,20 \\
Max & 20 & 1,54 & 1,16 & 34,71 & 34,09 & 10,28 & 11,69 & 9,39 & 9,95 \\
Prom & 20 & 1,46 & 1,00 & 34,51 & 34,04 & 8,80 & 10,42 & 8,58 & 9,78 \\
\hline
\end{tabular}
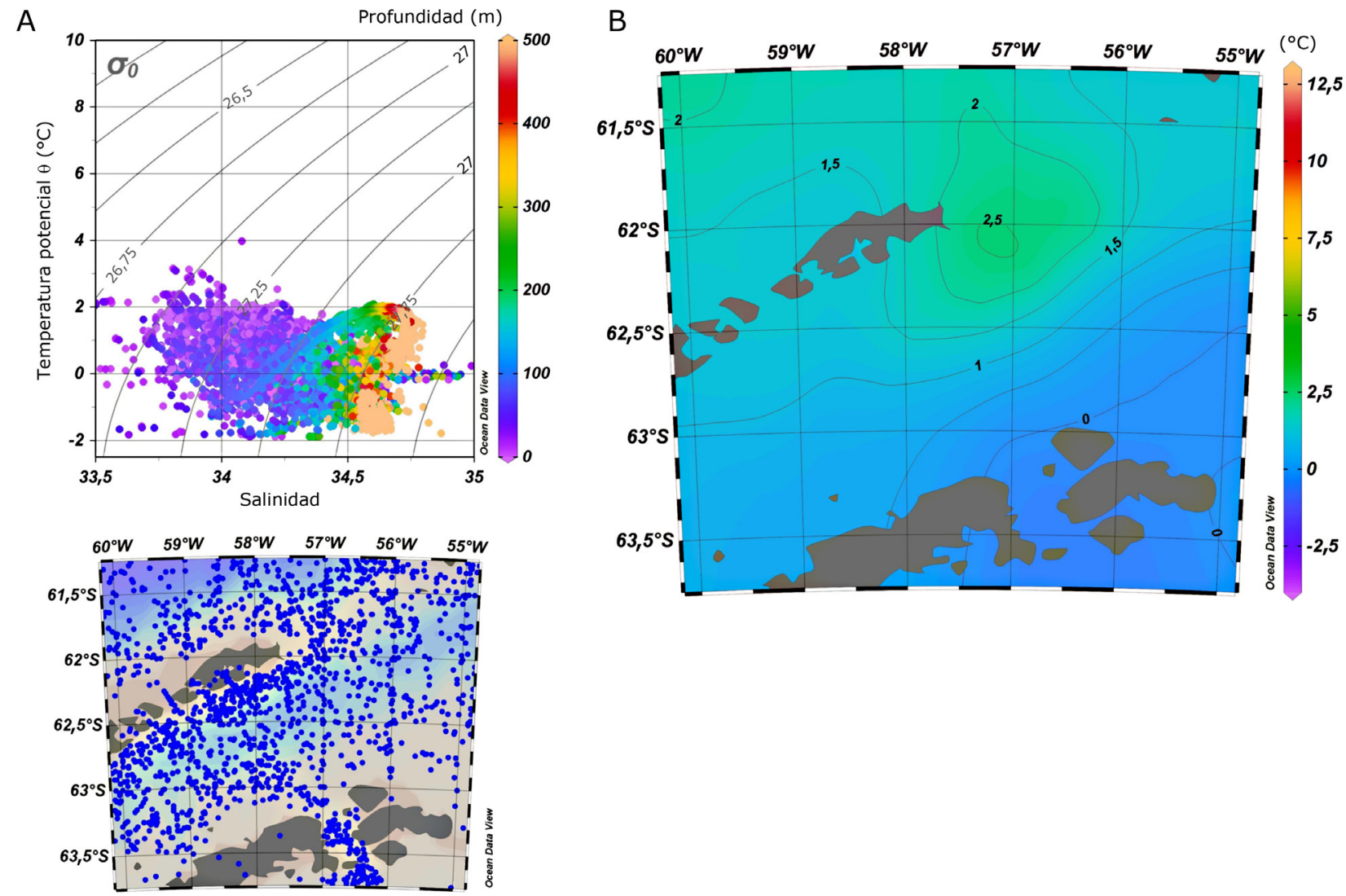

Figura 2. Condiciones oceanográficas entre las Islas Shetland del Sur y la Península Antártica para los veranos de los años 1970 a 2000. A) Masas de agua, B) Temperatura superficial del agua / Oceanographic conditions between southern Shetland Islands and the Antarctic peninsula for all summers of the years 1970 to 2000. A) Water masses, B) Sea surface temperature 
A
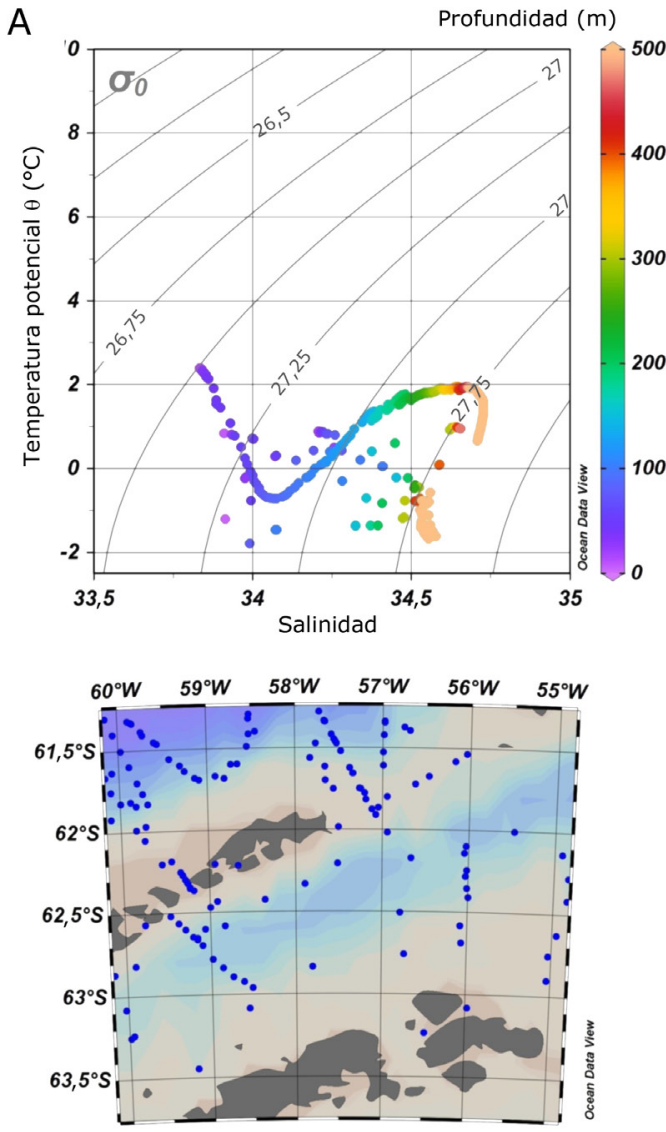

B



Figura 3. Condiciones oceanográficas entre las Islas Shetland del Sur y la Península Antártica para el verano del 2000. A) Masas de agua, B) Temperatura superficial del agua / Oceanographic conditions between southern Shetland Islands and the Antarctic peninsula for the summer 2000. A) Water masses, B) Sea surface temperature

\section{ESTRUCTURA DEL FITOPLANCTON}

Para el verano del 2012, la densidad del fitoplancton varió entre 2.940 células $\cdot \mathrm{L}^{-1}(\mathrm{E} 03-0 \mathrm{~m})$ y 409.010 células $\cdot \mathrm{L}^{-1}$ (E09-0 m). Los valores de abundancia fueron mayores en las estaciones E08 y E09. En el verano 2013, la densidad del fitoplancton varió entre 2.260 células $\cdot \mathrm{L}^{-1}(\mathrm{E} 04-0 \mathrm{~m}) \mathrm{y}$ 47.522 células $\cdot \mathrm{L}^{-1}(\mathrm{E} 11-20 \mathrm{~m})$. Las mayores concentraciones celulares se localizaron en las estaciones E10 y E11 que conforman la entrada de la ensenada, en superficie y $20 \mathrm{~m}$ de profundidad (Tabla 4).

El índice de diversidad ( $\left.\mathrm{H}^{\prime}\right)$ en general fluctuó entre 0,01 y 1,97 bits $\cdot$ célula $^{-1}$. Ambos veranos presentaron índices de diversidad bajos, mostrando una distribución bastante homogénea con valores entre 1 y 1,97 bits·célula ${ }^{-1}$ a excepción de las estaciones E08 y E09 (verano 2012) en donde los valores son muy bajos $\left(<0,1\right.$ bits·células $\left.{ }^{-1}\right)$ asociados a una baja riqueza de especies (menor de 11, ver Tabla 4).

El análisis de agrupamiento al $60 \%$ de similitud, define un solo grupo (G1) para ambos veranos, aglomerando a todas las estaciones de la ensenada. Entre las especies más frecuentes tenemos a Thalassiosira antarctica, Thalassiosira delicatula, Porosira glacialis y nanoflagelados (Figs. 4A y 4B).

\section{DISTRIBUCIÓN HORIZONTAL Y VERTICAL}

En el verano 2012, en la columna de agua ( 0,10 y $20 \mathrm{~m})$ se identificaron 42 especies, de las cuales 33 son diatomeas, 6 dinoflagelados y varios nanoflagelados. Los nanoflagelados fueron dominantes en los 3 estratos estudiados, con un promedio de $87 \%$ del total y las diatomeas con un $12 \%$ del total. Verticalmente el fitoplancton muestra una distribución bastante homogénea asociada a densidades menores de 18.000 células $\cdot \mathrm{L}^{-1}$, con excepción de pequeños parches en las estaciones E08 y E09 atribuido a los nanoflagelados (Tabla 1).

La ensenada presentó especies comunes de aguas antárticas como Corethron criophilum, Thalassiosira antarctica, Pseudo-nitzschia grupo delicatissima, Porosira glacialis, Haslea trompii, Fragilariopsis kerguelensis, Gyrodinium lachryma y Gymnodinium sp. (Tabla 5).

Algunas diatomeas bentónicas como Achnanthes sp., Gyrosigma fasciola, Gyrosigma sp. y Licmophora antartica estuvieron presentes en el primer transecto de la ensenada, colindante a los glaciales; mientras otras diatomeas bentónicas como Cocconeis placentula, Gomphonema margaritae, Navicula sp., Nitzschia sp. y Tropidoneis antarctica estuvieron presentes tanto en el primer como segundo transecto. 
Tabla 4. Número de especies, abundancia e índice de diversidad de Shannon-Weaver ( $\left.\mathbf{H}^{\prime}\right)$ registrados para los veranos 2012 y 2013 / Number of species, abundance and Shannon-Weaver index ( $\left.\mathrm{H}^{\prime}\right)$ recorded for the summers of 2012 and 2013

\begin{tabular}{|c|c|c|c|c|c|c|}
\hline Est/Prof & $\begin{array}{c}\mathrm{N}^{\circ} \text { especies } \\
(2012)\end{array}$ & Abundancia & Shannon $\left(\mathrm{H}^{\prime}\right)$ & $\begin{array}{c}\mathrm{N}^{\circ} \text { especies } \\
\quad(2013)\end{array}$ & Abundancia & Shannon $\left(\mathrm{H}^{\prime}\right)$ \\
\hline E1-0 & 19 & 46.502 & 1,49 & 11 & 6.598 & 1,68 \\
\hline E1-10 & 13 & 16.897 & 1,60 & 12 & 10.567 & 1,87 \\
\hline E1-20 & 15 & 33.979 & 1,62 & 14 & 8.700 & 1,45 \\
\hline E2-0 & 10 & 14.983 & 1,34 & 6 & 2.320 & 1,29 \\
\hline E2-10 & 20 & 30.504 & 1,63 & 14 & 8.458 & 1,51 \\
\hline $\mathrm{E} 2-20$ & 16 & 40.532 & 1,60 & 16 & 14.204 & 1,84 \\
\hline E3-0 & 10 & 2.940 & 1,50 & 12 & 9.317 & 1,97 \\
\hline E3-10 & 10 & 43.077 & 0,72 & 13 & 16.631 & 1,76 \\
\hline E3-20 & 13 & 23.940 & 1,40 & 12 & 11.324 & 1,21 \\
\hline E4-0 & 11 & 11.150 & 1,45 & 8 & 2.260 & 1,00 \\
\hline E4-10 & 21 & 9.177 & 1,52 & 10 & 14.872 & 1,65 \\
\hline E4-20 & 20 & 13.380 & 1,62 & 10 & 9.880 & 0,71 \\
\hline E5-0 & 13 & 23.431 & 0,86 & 12 & 8.268 & 1,45 \\
\hline E5-10 & 12 & 29.787 & 0,69 & 14 & 16.060 & 1,72 \\
\hline E6-0 & 15 & 7.789 & 1,90 & 8 & 7.187 & 1,76 \\
\hline E6-10 & 15 & 23.916 & 1,16 & 8 & 7.276 & 1,51 \\
\hline E6-20 & 16 & 52.857 & 1,05 & 12 & 22.028 & 1,54 \\
\hline E7-0 & 11 & 4.850 & 1,60 & 6 & 3.435 & 1,28 \\
\hline E7-10 & 16 & 23.180 & 1,30 & 12 & 11.116 & 1,86 \\
\hline E7-20 & 13 & 48.454 & 1,06 & 13 & 24.268 & 1,50 \\
\hline E8-0 & 7 & 32.110 & 0,10 & 7 & 5.078 & 1,53 \\
\hline E8-10 & 9 & 127.991 & 0,06 & 13 & 28.076 & 1,22 \\
\hline E8-20 & 11 & 367.471 & 0,02 & 12 & 21.780 & 1,54 \\
\hline E9-0 & 5 & 409.010 & 0,01 & 9 & 22.564 & 1,44 \\
\hline E9-10 & 9 & 165.187 & 0,07 & 10 & 7.291 & 1,65 \\
\hline E10-0 & 8 & 4.209 & 1,49 & 9 & 33.861 & 0,88 \\
\hline E10-10 & 10 & 10.275 & 1,01 & 9 & 25.076 & 1,45 \\
\hline E10-20 & 8 & 11.506 & 1,02 & 13 & 13.794 & 1,83 \\
\hline E11-0 & 9 & 4.609 & 1,33 & 6 & 5.822 & 1,16 \\
\hline E11-10 & 9 & 14.747 & 1,22 & 14 & 19.043 & 1,84 \\
\hline E11-20 & 7 & 3.715 & 1,22 & 12 & 47.522 & 0,77 \\
\hline
\end{tabular}

A

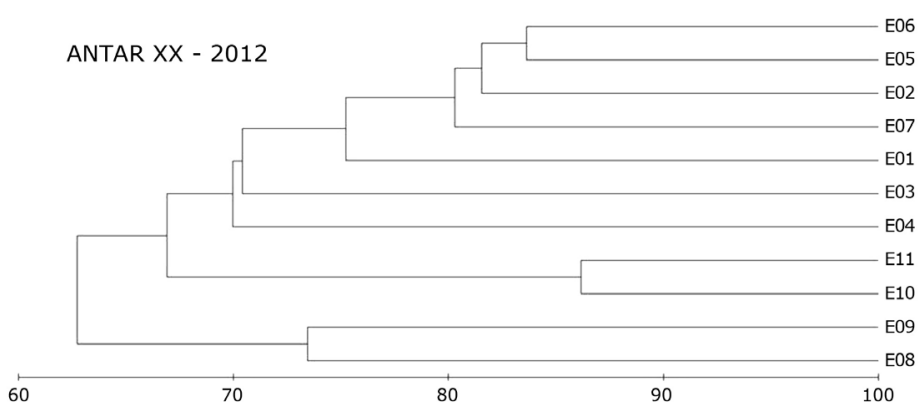

B

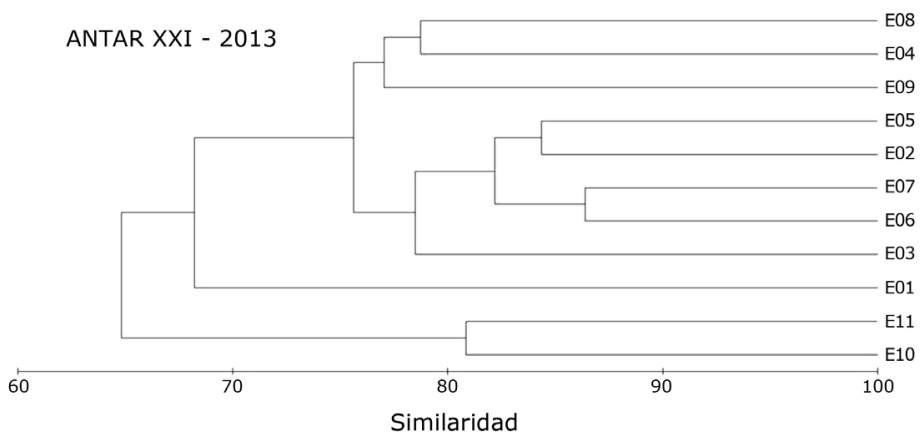

Figura 4. Similitud del fitoplancton durante las expediciones científicas ANTAR XX (verano austral 2012) y ANTAR XXI (verano austral 2013) en la ensenada Mackellar-Bahía Almirantazgo. A) Dendrograma de similitud (Índice Bray-Curtis), verano 2012. B) Dendrograma de similitud, verano 2013 / Similarity of the phytoplankton during the oceanographic cruises ANTAR XX (Austral summer 2012) and ANTAR XXI (Austral summer 2013) from the Mackellar inlet, Admiralty Bay. A) Dendrogram of similarity (Bray-Curtis index), summer 2012. B) Dendrogram of similarity, summer 2013 
En el verano 2013, en la columna de agua se identificaron 29 especies, de las cuales 23 fueron diatomeas, 4 dinoflagelados y varios nanoflagelados. Las diatomeas dominaron con un $56 \%$ del total. Verticalmente el fitoplancton estuvo dispuesto irregularmente, las mayores concentraciones de fitoplancton se localizaron en superficie y a $20 \mathrm{~m}$ de profundidad. Las diatomeas a $20 \mathrm{~m}$ llegaron a concentraciones celulares de 13.000 células $\cdot \mathrm{L}^{-1}$. Para los dinoflagelados en la E10-0 m y E11-20 m las concentraciones celulares llegaron a 26.363 y 39.587 células $\cdot \mathrm{L}^{-1}$ y para los nanoflagelados las abundancias tuvieron valores menores a 12.000 células $\cdot \mathrm{L}^{-1}$ (Tabla 2 ).

Las especies presentes en toda la ensenada fueron Thalassiosira antarctica, Thalassiosira delicatula, Pseudonitzschia grupo delicatissima, Porosira glacialis, Navicula sp., Gomphonema margaritae y Gyrodinium lachryma (Tabla 5).

Tabla 5. Lista de especies de diatomeas, dinoflagelados y nanoflagelados a tres profundidades durante los veranos 2012 y

2013 / List of species of diatoms, dinoflagellates and nanoflagellates at three depths during the summers of 2012 and 2013

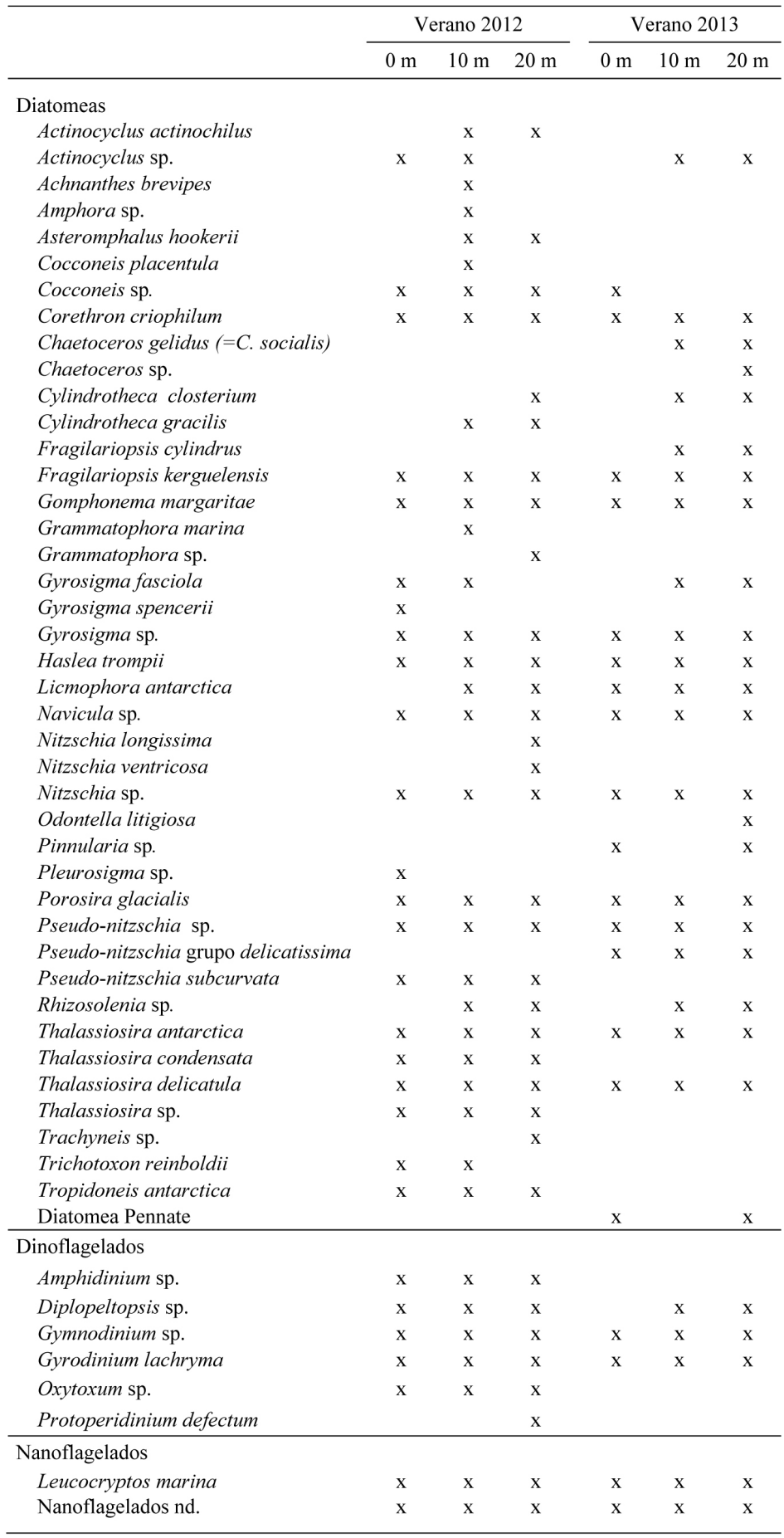

nd.: no determinado 
Las especies bentónicas como Cocconeis sp. y Gyrosigma sp. estuvieron presentes en el primer transecto, otras diatomeas bentónicas como Fragilariopsis kerguelensis y Nitzschia sp. en el primer y segundo transecto y cabe mencionar a Chaetoceros gelidus $(=C$. socialis $)$ en el primer y tercer transecto y Gymnodinium sp. en la entrada de la ensenada (tercer transecto).

La abundancia relativa de las principales especies para el 2012 muestra que esta comunidad estuvo dominada por nanoflagelados (86\%) (Fig. 5A). Para el 2013, la comunidad fitoplanctónica estuvo dominada por diatomeas céntricas de pequeño tamaño como Thalassiosira delicatula (36\%), Pseudo-nitzschia grupo delicatissima (9\%), Porosira glacialis (6\%), Thalassiosira antarctica (2\%), Navicula $\mathrm{sp}$. $(2 \%)$ y Chaetoceros gelidus $(=$ C. socialis) $(2 \%)$. Además de nanoflagelados $(20 \%)$ y el dinoflagelado Gymnodinium sp. (19\%) (Fig. 5B).

\section{FITOPLANCTON Y SU RELACIÓN CON LOS FACTORES AMBIENTALES}

El análisis de correspondencia canónica no mostró una asociación de las variables fisicoquímicas con el fitoplancton para el verano 2012, pero para el verano 2013 el análisis de correspondencia canónica mostró una asociación de algunas variables fisicoquímicas con los grupos fitoplanctónicos y con las abundancias de las estaciones a diferentes estratos. El eje 1 explica el 79\% de la variabilidad. Al eje 1 se asoció la salinidad con las estaciones que se ubican en el tercer transecto (E09 y E10); la temperatura se asoció más con las estaciones del primer (E03) y segundo transecto (E08 y E09). El eje 2 explica el 21\% de la variabilidad y mostró asociación entre el oxígeno y las diatomeas (Fig. 6).

A

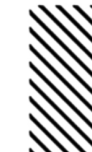

2,3

\section{2,1}

Thalassiosira

Thalassiosir delicatula

\section{5,4}

3,8

antarctica

Thalassiosira

sp.

Otros

sp.

B

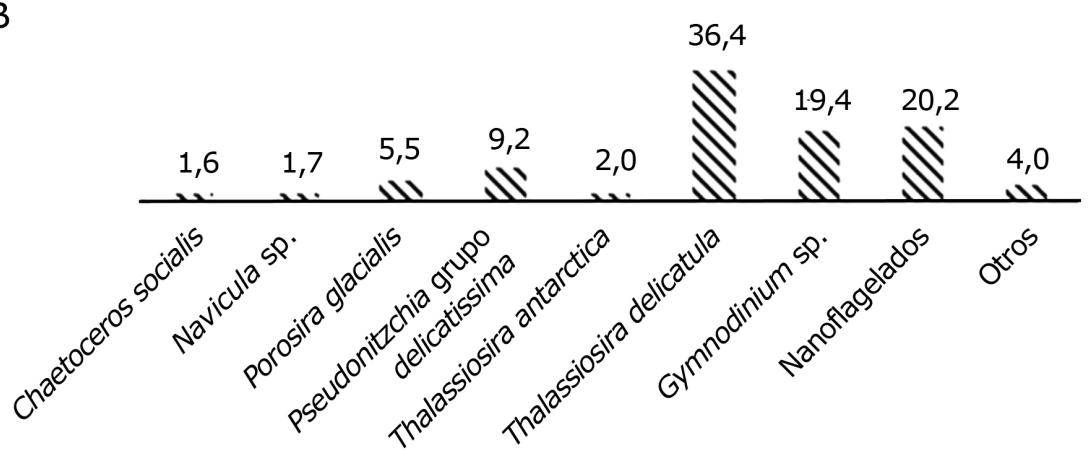

Figura 5. Abundancia relativa de especies de fitoplancton durante las expediciones cientificas: A) ANTAR XX (verano austral 2012) y B) ANTAR XXI (verano austral 2013) en la ensenada Mackellar-Bahía Almirantazgo / Relative abundance of phytoplankton species during the oceanographic cruises: A) ANTAR XX (Austral summer 2012) and B) ANTAR XXI (Austral summer 2013) from the Mackellar inlet, Admiralty Bay 


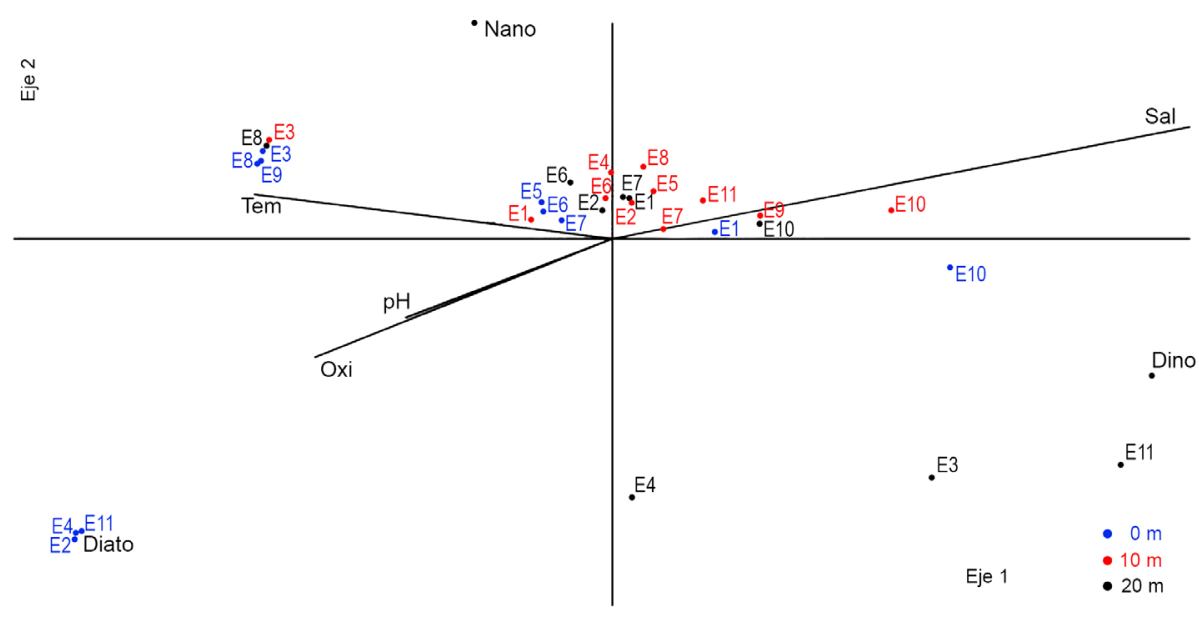

Figura 6. Análisis Canónico de Correspondencias (ACC). Diagrama de ordenación con grupos de fitoplancton (diatomeas, dinoflagelados y nanoflagelados), estaciones de muestreo $(\cdot)$ y variables ambientales: temperatura, salinidad, oxígeno y pH (líneas) en el espacio de los dos primeros ejes de ordenación. El eje horizontal representa el eje $\mathbf{1}$ y el eje vertical al eje $\mathbf{2}$ / Canonical correspondence analysis (ACC). Ordination diagram with phytoplankton groups (diatoms, dinoflagellates and nanoflagellates), sampling stations $(\cdot)$ and environmental variables: temperature, salinity, oxygen and $\mathrm{pH}$ (lines). Horizontal axis represents axis 1 and the vertical axis the axis 2

La prueba ANOSIM en los datos estandarizados de la abundancia de la raíz transformada indicó que todos los pares de estaciones fueron significativamente diferentes entre sí excepto H6 y H7, y aquí el nivel de significación fue sólo marginalmente $>5 \%$ (Tabla 6). Para los datos estandarizados de biomasa transformada de raíz doble, todos los pares de sitios fueron significativamente diferentes entre sí.

Para el verano 2013, el test de ANOSIM con los datos de abundancia estandarizados indica que dos pares de profundidades ( 0 y $10 \mathrm{~m}$ de profundidad y 0 y $20 \mathrm{~m}$ de profundidad) fueron significativamente diferentes entre sí $(P$ $<0,05$ ) el valor de $\mathrm{R}$ fue de 0,179 (Tabla 6). Estos resultados fueron respaldados por el análisis de SIMPER identificando a los nanoflagelados (18-33\%), Thalassiosira delicatula (20-32\%) y Gymnodinium sp. (16\%) como las principales especies que contribuyeron con la disimilitud (53-59\%) en la variación de las diferentes profundidades (Tabla 7).
Tabla 6. Análisis ANOSIM, valores de R y nivel de significancia $(P<$ 0,05 ) basado en la abundancia de las especies fitoplanctónicas en comparaciones realizadas entre los periodos de estudio / ANOSIM analysis, $\mathrm{R}$ values and significance level $(P<0.05)$ based on the abundance of the phytoplanktonic species comparing study periods

\begin{tabular}{ccc}
\hline Periodos & $\mathrm{R}$ & $\begin{array}{c}\text { Nivel de significancia } \\
(P<0,05)\end{array}$ \\
\hline Verano 2012 & 0,0484 & 0,1407 \\
Verano 2013 & 0,179 & 0,0006 \\
\hline
\end{tabular}

Tabla 7. Valores del Análisis SIMPER para la contribución de las especies/taxones para el verano 2013 a diferentes profundidades / Values of the SIMPER analysis for species or taxa contributions for summer 2013 at different depths

\begin{tabular}{lccccc}
\hline \multicolumn{1}{c}{ Taxon } & Abun. Prom. $(0 \mathrm{~m})$ & Abun. Prom. $(10 \mathrm{~m})$ & Diss. Prom. & \% Contrib. & \% Acum. \\
\hline Nanoflagelados & 992 & 4.270 & 17,92 & 33,81 & 33,81 \\
Thalassiosira delicatula & 3.170 & 3.490 & 11,05 & 20,84 & 54,64 \\
Gymnodinium sp. & 2.410 & 874 & 8,665 & 16,34 & 70,99 \\
\hline \multicolumn{1}{c}{ Taxon } & Abun. Prom. $(0 \mathrm{~m})$ & Abun. Prom. $(20 \mathrm{~m})$ & Diss. Prom. & $\%$ Contrib. & $\%$ Acum. \\
\hline Thalassiosira delicatula & 3.170 & 6.080 & 19,32 & 32,45 & 32,45 \\
Nanoflagelados & 992 & 2.550 & 11,02 & 18,51 & 50,96 \\
Gymnodinium sp. & 2.410 & 4.310 & 9,851 & 16,54 & 67,5 \\
\hline
\end{tabular}

Abun Prom: Promedio de abundancia, Diss Prom: Promedio de disimilaridad, \% Contrib: Porcentaje de contribución, \% Acum: Porcentaje Acumulativo 
Para el verano 2012, la prueba ANOSIM en los datos estandarizados de la abundancia no mostró diferencias significativas entre las profundidades $(P>0,05)$. El valor de $\mathrm{R}$ fue de 0,0484 (Tabla 6). El análisis SIMPER, para evaluar a los grupos fitoplanctónicos que contribuyeron más a la disimilitud entre los dos periodos de estudio, se muestra en la Tabla 8. Se evidencia que la mayor contribución (64\%) estuvo dada por los nanoflagelados.

\section{DisCUSIón}

\section{HidRografía Y CONDICIONES OCEANOGRÁFICAS}

Durante el 2012 y 2013 se presentaron temperaturas superficiales de mar (TSM) en la ensenada Mackellar en un rango de diferencia entre 0,22 a $0,41^{\circ} \mathrm{C}$, respectivamente, mientras que en los alrededores de la bahía Almirantazgo los valores de TSM varían entre $0,75 \pm 0,42{ }^{\circ} \mathrm{C}$ para el comienzo del verano 2011 y $1,61 \pm 0,15{ }^{\circ} \mathrm{C}$ para finales del verano 2011 (Tenorio et al. 2012). Cabe señalar, que los valores de salinidad en los alrededores de la Isla Rey Jorge presentaron signos de derretimiento del glaciar, lo cual se evidenció en la relación encontrada entre la gran cantidad de partículas suspendidas y los eventos de mortalidad de krill entre 2003 a 2012 (Fuentes et al. 2016). El incremento de TSM en los alrededores de la Isla Rey Jorge durante 1970 a 1999, y sobre todo durante el 2010 (Figs. 2 y 3) muestra la señal de calentamiento que sufre esta zona de la Antártica, en especial las pequeñas bahías, canales y ensenadas de la Isla Rey Jorge, afectando gradualmente el ecosistema de los polos (Smetacek \& Nicol 2005).

En el estudio realizado por Sánchez et al. (2013) en la bahía Almirantazgo, la comunidad fitoplanctónica se caracterizó por la dominancia de la fitoflagelados ND asociados a temperaturas promedio de $1,11^{\circ} \mathrm{C}$ y salinidad promedio de 33,86 ; en cambio en nuestro estudio del verano
2013 la comunidad fitoplanctónica se ha caracterizado por la abundancia de diatomeas (59\%), dinoflagelados (20\%) y nanoflagelados $(20 \%)$ asociados a temperatura promedio de $1,27^{\circ} \mathrm{C}$ y salinidad promedio de 33,9 ; la diferencia en la dominancia se puede deber a una alta concentración de nutrientes en zonas más someras que propicia el desarrollo de estos grupos fitoplanctónicos, en cambio a lo largo de la bahía Almirantazgo la prevalencia de los fitoflagelados se puede atribuir a la dinámica de las condiciones hidrográficas características como por ejemplo: los vientos y la estabilidad en la columna de agua que propiciaría la dominancia de este grupo.

\section{COMPOSICIÓN DEL FITOPLANCTON}

En ambos periodos de estudio la composición de especies de diatomeas fue similar presentándose diatomeas céntricas de pequeño tamaño como Thalassiosira antarctica, Thalassiosira delicatula, Porosira glacialis y la diatomea formadora de colonias Pseudo-nitzschia grupo delicatissima, además del dinoflagelado común en aguas antárticas Gyrodinium lachryma. Estudios realizados por Sánchez et al. (2013) en el estrecho de Bransfield en el verano 2013 confirman la presencia de especies de los géneros Pseudo-nitzschia y Thalassiosira (Tabla 5).

\section{DISTRIBUCIÓN DEL FITOPLANCTON}

Durante el primer periodo de estudio (2012) los nanoflagelados fueron numéricamente el grupo más abundante, muy similar a lo reportado por Kopczyńska (1980, 1992, 2008) y Annett et al. (2010). Otros estudios reportaron para el segundo periodo de estudio (2013) que la dominancia del fitoplancton cambió, ya que fueron las diatomeas "centrales" de pequeño tamaño las que dominaron en número.

Tabla 8. Valores del Análisis SIMPER para la contribución de los grupos fitoplanctónicos entre el verano 2012 y 2013 / Values of the SIMPER analysis for contributions of taxonomic groups between summer 2012 and 2013

\begin{tabular}{lccccc}
\hline \multicolumn{1}{c}{ Taxón } & $\begin{array}{c}\text { Abun. Prom. } \\
(2012)\end{array}$ & $\begin{array}{c}\text { Abun. Prom. } \\
(2013)\end{array}$ & Diss. Prom. & \% Contrib. & \% Acum. \\
\hline Nanoflagelados & 45.700 & 2.550 & 38,62 & 64,41 & 64,41 \\
Diatomeas & 7.130 & 6.760 & 16,31 & 27,19 & 91,6 \\
Dinoflagelados & 445 & 2.540 & 5,04 & 8,404 & 100 \\
\hline
\end{tabular}

Abun Prom: Promedio de abundancia, Diss Prom: Promedio de disimilaridad, \% Contrib: Porcentaje de contribución, \% Acum: Porcentaje Acumulativo 
Durante el verano 2012, la prevalencia en dominancia de los nanoflagelados sobre las diatomeas se puede atribuir a la estabilidad en la columna de agua originando un alto desarrollo de microalgas de los grupos taxonómicos Prasinophyceae, Cryptophyta o Haptophyta, en aguas con bajas abundancias de diatomeas, caso similar se dio en el periodo de estudio de 1996-98 y 2003-05 en la bahía Almirantazgo (Kopczynska 2008). Esta estabilidad en la columna de agua puede deberse también a una distribución casi uniforme de la temperatura y salinidad en la columna de agua. Según Pruszak (1980), los pequeños cambios en la salinidad, se produce principalmente por aporte de agua dulce de los glaciares. En el verano 2012, la salinidad promedio en cada estrato fue bastante uniforme, lo que podría favorecer a las poblaciones de nanoflagelados, en cambio para el verano 2013 la salinidad fue mayor a 20 m $(34,04)$ comparado a nivel superficial, lo cual indicaría un mayor aporte de agua dulce de los glaciales los cuales podrían favorecer más a las poblaciones de diatomeas y dinoflagelados que a los nanoflagelados.

La turbulencia favorece el mantenimiento de las poblaciones fitoplanctónicas en las zonas más superficiales, contrariamente a lo que sucede en condiciones de estabilidad en la que se configura una distribución vertical del fitoplancton caracterizada por altas densidades de células en profundidad debido al hundimiento de las poblaciones de diatomeas (Avaria \& Muñoz 1982), lo que se puede corroborar con nuestros resultados en donde las densidades de diatomeas a $20 \mathrm{~m}$ fueron relativamente mayores que en superficie para el 2013 cuya densidad de diatomeas a 20 $\mathrm{m}$ fue más del doble que en superficie (59.591 células $\cdot \mathrm{L}^{-1}$ en superficie y 106.102 células $\cdot \mathrm{L}^{-1}$ a $20 \mathrm{~m}$ ). Además de la turbulencia las diatomeas se pueden ver favorecidas por la limitación de la luz, cuando la fotosíntesis relativa se representa frente a la intensidad de la luz, los organismos de hielo y el plancton aumentan en productividad hasta aproximadamente $18 \mathrm{klx}$ y son algo inhibidos por mayores intensidades a 29 klx (Burkholder \& Mandelli 1965), las cuales podrían darse en los primeros metros de profundidad.

En la Antártica se ha descrito un grupo especial de microalgas conocidas como "diatomeas bentónicas" que viven en el fondo, pegadas a las piedras o sedimentos. Ellas son capaces de hacer fotosíntesis con niveles de luz muy bajos (invierno). Esta capacidad se ha llamado foto-adaptación (Uribe 1982). En este estudio, para el verano 2012 se encontraron diatomeas bentónicas como Achnanthes sp., Gyrosigma fasciola, Gyrosigma sp. y Licmophora antartica y para el verano 2013 se encontraron las especies Cocconeis sp. y Gyrosigma sp. presentes en toda la columna de agua del primer transecto, colindante a los glaciales de hielo, lo cual podría indicar que estas diatomeas están capacitadas para vivir debajo de las capas de hielo marino y favorecidas por los altos niveles de luz durante todo el día en la columna de agua, característica propia de la época de verano.
Ambos veranos presentaron bajos valores en los índices de diversidad, siendo menores de 1,32 bits·célula ${ }^{-1}$, lo cual es característico de una comunidad típica de la primera fase de la sucesión ecológica (Margalef 1958); similares características fueron reportadas por Antonietti (1989), Gómez (1991), Delgado (1999) y Sánchez \& Villanueva (2001).

\section{Agradecimientos}

Al Proyecto: "Biodiversidad del macrobentos y plancton marino en la ensenada Mackellar y la Bahia Almirantazgo, Isla Rey Jorge - Antartida" de la Dirección de Asuntos Antárticos, Ministerio de Relaciones Exteriores del Perú por el financiamiento del trabajo que constituye parte de la Tesis de Maestría: 'Variación espacial e interanual del fitoplancton durante los veranos australes de la ensenada MackellarIsla Rey Jorge, Antártica: 2008-2013', de la Universidad Nacional Mayor de San Marcos. Agradecemos a la Dra. Noemí Ochoa por la confirmación en la identificación de las especies, a todos los expedicionarios que colaboraron con el muestreo y a Bernabé Moreno por la elaboración del mapa.

\section{LiTERATURA CITADA}

Annett AL, DS Carson, X Crosta, A Clarke \& RS Ganeshram. 2010. Seasonal progression of diatom assemblages in surface waters of Ryder Bay, Antarctica. Polar Biology 33: 13-29.

Antonietti E. 1989. Microplancton del Estrecho de Bransfield. Verano de 1989 (Perú ANTAR II). Informe Científico de la Segunda Expedición Peruana a la Antártida, Lima, pp. 9-44.

Avaria S \& P Muñoz. 1992. Producción actual, biomasa y composición específica del fitoplancton de la Bahía de Valparaíso en 1979. Revista de Biología Marina 18(2): 129-157.

Balech E. 1944. Plancton de Lennox y Cabo de Hornos. Revista de la Sociedad Argentina de Ciencias Naturales (Physis) 19(54): 29-76.

Balech E. 1976. Clave ilustrada de dinoflagelados antárticos. Instituto Antártico Argentino, Dirección Nacional del Antártico, Pub. IAA 11: 1-98.

Bonicelli J, D López, N Ochoa \& R Shreeve. 2008. Estructura comunitaria del zooplancton asociada con el fitoplancton y las masas de agua del Estrecho de Bransfield y La Isla Elefante durante el verano austral del 2006. Ecología Aplicada 7(1-2): 159-164.

Burkholder PR \& EF Mandelli. 1965. Productivity of microalgae in Antarctic sea ice. Science 149(3686): 872-874.

Catewicz Z. 1984. Variability of water flow in Ezcurra Inlet. Oceanologia 15: 73-95.

Clarke KR \& RN Gorley. 2006. PRIMER 6. User Manual/ Tutorial. Primer-E Ltd, Plymouth.

Delgado E. 1999. Fitoplancton del Estrecho de Bransfield e Isla Elefante durante el verano austral 1998. Resultados del programa de Investigación Antártica del Instituto del Mar del Perú Verano Austral 1998, Perú ANTAR IX, Segunda parte, Crucero BIC Humboldt 9801, Informe IMARPE 145: 4-22.

Frenguelli J. 1943. Diatomeas de las orcadas del Sur. Revista del Museo de la Plata, Tomo 5, Botánica 21: 221-277. 
Frenguelli J \& HA Orlando. 1958. Diatomeas y silicoflagelados del sector antártico sudamericano, 191 pp. Instituto Antártico Argentino, Buenos Aires.

Fuentes V, G Alurralde, B Meyer, GE Aguirre, A Canepa, AC Wolfl, HC Hass, GN Williams \& IR Schloss. 2016. Glacial melting: an overlooked threat to Antarctic krill. Scientific Reports 6(27234). < doi: 10.1038/srep27234>.

García-Muñoz C, LM Lubián, CM García, A Marrero, P Sangrà \& M Vernet. 2013. A mesoscale study of phytoplankton assemblages around the South Shetland Islands (Antarctica). Polar Biology 36: 1107-1123.

Garibotti IA, M Vernet \& ME Ferrario. 2005. Annually recurrent phytoplanktonic assemblages during summer in the seasonal ice zone west of the Antarctic Peninsula (Southern Ocean). Deep Sea Research I 52: 1823-1841.

Gili J, O Cavadonga, R Joandomènec, P López \& WE Arntz. 2000. La vida en los fondos antárticos. Investigación y Ciencia 290: 64-74.

Gómez O. 1991. Microplancton del Estrecho de Bransfield durante el verano de 1991. Informes Científicos de la Tercera Expedición del Perú a la Antártida, pp. 109-164. Comisión Nacional de Asuntos Antárticos (CONAAN), Lima.

Hammer Ø, DAT Harper \& PD Ryan. 2001. PAST: Paleontological statistics software package for education and data analysis. Palaeontologia Electronica 4(1): 1-9. $<$ http://palaeo-electronica.org/2001_1/past/issue1_01.htm>

Hammer Ø, D Harper \& PD Ryan. 2013. PAST: Palaeontological Statistics, Ver. 3.14. Natural History Museum, Universidad de Oslo, Oslo. $<$ https://folk.uio.no/ohammer $>$

Hasle GR. 1969. An analysis of the phytoplankton of the Pacific Southern Ocean: abundance, composition and distribution during the Brategg expedition 1947/8. Hvalradetes Skrifter 52: $1-168$.

Hasle GR \& EE Syvertsen. 1997. Marine diatoms. In: Tomas C (ed). Identifying marine phytoplankton, pp. 5-385. Academic Press, San Diego.

Kopczyńska EE. 1980. Small scale vertical distribution of phytoplankton in Ezcurra Inlet, Admiralty Bay, South Shetland Islands. Polish Polar Research 1: 77-96.

Kopczyńska EE. 1981. Periodicity and composition of summer phytoplankton in Ezcurra Inlet, Admiralty Bay, South Shetland Islands. Polish Polar Research, 2(3-4): 55-70.

Kopczyńska EE. 1992. Dominance of microflagellates over diatoms in the Antarctic areas of deep vertical mixing and krill concentrations. Journal of Plankton Research 14: 1031-1054.

Kopczyńska EE. 1996. Annual study of the phytoplankton in Admiralty Bay, King George Island, Antarctica. Polish Polar Research 17(1-2): 151-164.

Kopczyńska EE. 2008. Phytoplankton variability in Admiralty Bay, King George Island, South Shetland Islands: six years of monitoring. Polish Polar Research 29(2): 117-139.

Lund J, C Kipling \& D LeCren. 1958. The inverted microscope of estimating algal numbers and the statistical basis of estimations by counting. Hydrobiologia 11: 143-170.

Margalef R. 1958. Temporal succession and spatial heterogeneity in natural phytoplankton. In: Buzzati-Traverso AA (ed). Perspectives in marine biology, pp. 232-329. University of California Press, Berkeley.
Medlin LK \& J Priddle. 1990. Polar marine diatoms, 214 pp. British Antarctic Survey, Natural Environmental Council, Cambridge.

Priddle J \& G Fryxell. 1985. Handbook of the common plankton diatoms of the Southern Ocean: Centrales except the genus Thalassiosira, 159 pp. British Antarctic Survey, Natural Environmental Council, Cambridge.

Pruszak Z. 1980. Current circulation in the waters of Admiralty Bay (region of Arctowski Station on King George Island). Polish Polar Research 1: 55-74.

Rakusa-Suszczewski S. 1980. Environmental conditions and the functioning of Admiralty Bay (South Shetland Islands) as part of the near-shore Antarctic ecosystem. Polish Polar Research 1: 11-27.

Rakusa-Suszczewski S. 1993. Hydrography and hydrochemistry. In: Rakusa-Suszczewski S (ed). The maritime antarctic coastal ecosystem of Admiralty Bay, pp. 32-34. Polish Academy of Sciences, Warszaw.

Rakusa-Suszczewski S. 1996. Spatial and seasonal variability of temperature and salinity in the Bransfield Strait and Admiralty Bay, Antarctica. Polish Polar Research 17: 29-42.

Rakusa-Suszczewski S \& A Kidawa. 1997. Variability of the South Shetland Islands Geoeco- system. Geographia Polonica 70: 65-72.

Robakiewicz M \& S Rakusa-Suszczewski. 1999. Application of $3 \mathrm{D}$ circulation model to Admiralty Bay, King George Island, Antarctica. Polish Polar Research 20: 43-58.

Round FE, RM Crawford \& DG Mann. 1990. The diatoms: Biology \& morphology of the genera, 747 pp. Cambridge University Press, Cambridge.

Sánchez S \& P Villanueva. 2001. Composición del fitoplancton en el estrecho de Bransfield e Isla Elefante durante el verano austral de 1999. Revista Peruana de Biología 8(1): 23-37.

Sánchez S, P Villanueva \& E Delgado. 2013. Patrón de la distribución espacial del fitoplancton en el Estrecho de Bransfield durante el verano austral 2013 Perú-ANTAR XXI. En: Salazar-Cespedes CM (ed). ANTAR XXI. Informe Final. Investigaciones Científicas Antárticas del IMARPE en el Estrecho de Bransfield, Bahía Almirantazgo y Ensenada Mackellar, pp. 30-44. IMARPE, Callao.

Sayed Z. 2005. History and evolution of primary productivity studies of the Southern Ocean. Polar Biology 28: 423-438.

Secretaría del Trabajo Antártico. 1996. Plan de gestión de la zona Antártica especialmente administrada $\mathrm{N}^{\circ} 1$. Bahía del Almirantazgo (Bahía Lasserre), Isla Rey Jorge (Isla 25 De Mayo). Medida 2 - anexo, pp. 177-209. Partes consultivas del tratado Antártico en la XX RCTA, Buenos Aires, Argentina. $<$ www.ats.aq/documents/recatt/Att338_s.pdf >

Shannon C \& W Weaver. 1963. The mathematical theory of communication, $125 \mathrm{pp}$. University of Illinois Press, Urbana.

Smetacek V \& S Nicol. 2005. Polar ocean ecosystems in a changing world. Nature 437(7057): 362-368.

Sournia A, JR Grall \& G Jacques. 1979. Plankton diatoms and dinoflagellates along a meridian transect in the Southern Indian Ocean (campagne "Antiprod I" du Marion-dufresne, mars 1977). Botanica Marina 22: 183-198.

Steyeart J. 1973. Distribution of plankton diatoms along an African-Antarctic transect. Investigaciones Pesqueras 37: 295-328. 
Szafranski J \& M Lipski. 1982. Characteristics of water temperature and salinity in Admiralty Bay (King George Island) during austral summer 1978-1979. Polish Polar Research 3: 7-24.

Tenorio MMB, JJ Barrera-Alba \& D Rivera. 2012. Plankton structure of the shallow coastal zone at Admiralty bay, King George Island, West Antarctic Peninsula (WAP): chlorophyll biomass and size-fractionated chlorophyll during Austral summer 2010/2011. INCT-APA Annual Activity Report 10. $<$ doi: 10.4322/apa.2014.105>.

Torres G, C Palacios, T Calderón \& S Recalde. 2006. Interacción del fitoplancton y zooplancton y sus condiciones oceanográficas durante el verano austral 2004 (Isla Greenwich-Antártica). Revista Tecnológica-ESPOL 19(1): 153-160.

Turner J, SR Colwell, GJ Marshall, TA lachlan-Cope, AM Carleton, PD Jones, V Lagun, PA Reid \& S Iagovkina. 2005. Antarctic climate change during the last 50 years. International Journal of Climatology 25: 279-294.
Uribe E. 1982. Influence of the phytoplankton and primary production of the Antarctic waters in relationship with the distribution and behaviour of krill. INACH, Scientific Series 28: 147-163.

Vanzan M, JJ Barrera-Alba, MM Barboza \& D Rivera. 2015. Picoplankton and nanoplankton variability in an Antarctic shallow coastal zone (Admiralty Bay) during the austral summer of 2010/2011. Polar Biology 38(8): 1267-1284.

Wasilowska A, E Kopczynska \& M Rzepecki. 2015. Temporal and spatial variation of phytoplankton in Admiralty Bay, South Shetlands: the dynamics of summer blooms shown by pigment and light microscopy analysis. Polar Biology 38: $1249-1265$. 